\title{
Fractional Excretion of Sodium
}

National Cancer Institute

\section{Source}

National Cancer Institute. Fractional Excretion of Sodium. NCI Thesaurus. Code C107435.

The percentage of the total sodium filtered by the kidney that is excreted in the urine.

The fractional excretion is calculated by multiplying urinary sodium concentration by the plasma creatinine concentration, then dividing by the urinary creatinine concentration multiplied by the plasma sodium concentration. The resulting ratio is multiplied by 100 . 